\title{
Columnar self-assembly of a 3D-persulfurated coronene asterisk. The dominant role of aryl-sulfur bonds $\dagger \ddagger$
}

\author{
Peter Kowalzik, ${ }^{a}$ Silke Rathgeber, ${ }^{b}$ Silvia Karthäuser,,$\S^{* a}$ Rainer Waser, ${ }^{a}$ \\ Nicolas Schnaebele, ${ }^{c}$ Jean-Manuel Raimundo ${ }^{c d}$ and Marc Gingras $\uparrow^{\top} * c d$ \\ Received (in Montpellier, France) 1st June 2011, Accepted 14th September 2011 \\ DOI: $10.1039 / \mathrm{c} 1 \mathrm{nj} 20472 \mathrm{f}$
}

The synthesis, adsorption behavior, surface structure, and the charge transport properties of a persulfurated coronene asterisk with a 3D-polyaromatic system, namely dodecakis(phenylthio)coronene (DPTC), deposited on $\mathrm{HOPG}(0001)$ and $\mathrm{Au}(111)$ surfaces, are investigated by means of scanning tunneling microscopy (STM) and spectroscopy (STS). DPTC molecules adsorbed on HOPG(0001) show an orbital mediated tunneling through mainly undisturbed frontier molecular states. DPTC molecules self-assemble on $\mathrm{Au}(111)$ into a highly ordered $\pi$-stacked columnar "edge-on pattern. The columnar stacking is a gold surface mediated process, as ascertained by fluorescence correlation spectroscopy (FCS). DPTC was monomeric in the precursor solution before assembly. The tunneling spectra of ordered DPTC stacks on $\mathrm{Au}(111)$ show an energetic splitting of the frontier molecular states, indicating orbital overlap and supramolecular $\pi-\pi$ interactions of adjacent molecules. DPTCs are sufficiently flexible to facilitate dense 1D stacks. The multiple aryl-sulfur bonds play a dominant role in the modulation of the self-assembly properties of the coronenes which in turn affect their electronic properties. Our results encourage further applications in dendrimer chemistry toward molecular electronics, by using the functionalized coronene core and its multidirectional 3D properties.

\section{Introduction}

Electronic devices based on single organic molecules, oligomers, polymers, or organic thin films present alternatives to inorganic semiconductor devices. Research focuses on promising applications like organic light emitting diodes, solar cells, field effect transistors, and molecular switches. ${ }^{1-4}$ In particular, the use of disk-type polycyclic aromatic hydrocarbons (PAHs) as a basic

\footnotetext{
${ }^{a}$ Peter Grünberg Institut and JARA-FIT, Forschungszentrum Jülich GmbH, 52428 Jülich, Germany.E-mail: s.karthaeuser@fz-juelich.de; Fax: + 49 2461612550; Tel: + 492461614015

${ }^{b}$ University Koblenz-Landau, Institute for Natural Sciences, 55128 Koblenz, Germany. E-mail: rathgeber@uni-koblenz, Fax: + 49 2612872353; Tel: +492612871002353

${ }^{c}$ Former address, University of Nice-Sophia Antipolis,

28 Ave. Valrose, 06103 Nice Cedex 2, France

${ }^{d}$ CNRS UPR 3118 CINAM and Aix-Marseille University, 163 Ave. de Luminy, 13288 Marseille Cedex 09, France. E-mail: marc.gingras@univmed.fr; Fax: +33 491829155; Tel: +33491829155

$\dagger$ This article is part of the themed issue Dendrimers II, guest-edited by Jean-Pierre Majoral.

$\ddagger$ Electronic supplementary information (ESI) available: STM image and additional STS data for DPTC on HOPG(0001) at $T=77 \mathrm{~K}$. XPS data of the DPTC monolayer on Au(111). Details of the FCS experimental procedure and determination of the hydrodynamic radius of DPTC from expressions that take hydrodynamic interactions in FCS measurements into account. See DOI: $10.1039 / \mathrm{clnj} 20472 \mathrm{f}$

$\S$ Corresponding author for STM and electron transport studies.

- Corresponding author for organic synthesis, sulfur and supramolecular chemistry.
}

molecular unit is regarded to be an advantageous approach for the build up of electronic components. For instance, columnar phases of discotic materials can be utilized as charge transport channels with high charge carrier mobilities. ${ }^{5-8}$ The electronic properties of PAH derivatives may be specifically tuned by modifying the size of the aromatic $\pi$-system, the functional substituents and their electronic contribution. The performance of devices made from single molecules or molecular assemblies will largely depend on the supramolecular order as well as on the interfaces between molecular elements, supporting substrates, connecting electrodes, and on the supramolecular order.

In this study, we focus on the most simple molecular asterisk with a coronene core incorporating twelve non-functionalized arylthio groups, namely dodecakis(phenylthio)coronene (DPTC), and its propensity to make new supramolecular columnar selfassemblies on gold surfaces. In spite of a tremendous number of persulfurated aromatic derivatives in the literature, surface studies by STM/STS were performed only recently by some of us, ${ }^{9}$ even if the first member of this family of compounds was reported in $1957 .{ }^{10}$ Aromatic thioether (sulfide) groups are largely unexplored as an anchoring unit to substrates. ${ }^{11,12}$ Investigation on the adsorption of aromatic thioethers could be advantageous, because they are known to show a better resistance towards oxidation, compared to thiols ( $\mathrm{R}-\mathrm{SH})$, dialkyl sulfides (R-S-R), and disulfides (R-SS-R).

Phenylthio-substitution of the PAH core leads to highly interesting opto-electronic properties. UV-visible and 
spectroelectrochemistry revealed an effective electronic delocalization throughout the whole molecular system and a greater stabilization of charged species in DPTC compared to non-substituted PAHs. ${ }^{13-16}$ In the case of persulfurated coronenes, two consecutive single-electron reduction waves are observed and the sulfur stabilization of the resulting species is estimated to be $1 \mathrm{~V}$ for each reduction, compared to coronene itself. Aryl-S bonds are thus dominating several properties of PAH derivatives.

Combined scanning tunneling microscopy (STM) and spectroscopy (STS) ${ }^{17,18}$ allow the direct correlation of the charge transport properties of molecules according to the molecular structures. This technique is in particular sensitive to changes of the electronic structure of molecular assemblies, like $\pi-\pi$ - or charge-transfer interactions. ${ }^{19-24}$ Here, we report on the results based on the properties of persulfurated PAHs obtained by STM/STS investigations combined to FCS and XPS studies, the charge transport properties and the selfassembly of DPTC deposited on Highly Oriented Pyrolytic Graphite HOPG(0001) and on gold Au(111) surfaces.

\section{Results and discussions}

\section{Structural characteristics by STM}

The surface morphology of DPTC (see Fig. 1) adsorbed on HOPG(0001) and $\mathrm{Au}(111)$ surfaces has been investigated using UHV-STM. For DPTC monolayers deposited on HOPG(0001), no ordered DPTCs self-assembly was observed at room temperature (RT). It is assumed that DPTC is only weakly physisorbed and the residual mobility of the molecules prevents imaging with STM. De Feyter and De Schryver ${ }^{32}$ also reported that in the case of weak adsorbate-substrate interactions, STM imaging often becomes impossible. At $77 \mathrm{~K}$, the STM images show a molecular layer with features of single molecules (ESI $\ddagger$ ).

In contrast, ordered, short range columnar self-assemblies are obtained on $\mathrm{Au}(111)$. The surface morphology of DPTC adsorbed on $\mathrm{Au}(111)$ is presented in Fig. 2(a). The STM image (recorded at RT) shows a disordered monolayer with some bright spots of nearly identical size and most interestingly, a well-ordered phase consisting of parallel columns. The fraction of the surface covered with this columnar structure amounts to approximately $10 \%$ in the case of a freshly prepared sample. The number of ordered domains and the domain sizes increases during storage in the UHV chamber at RT. Molecules in the

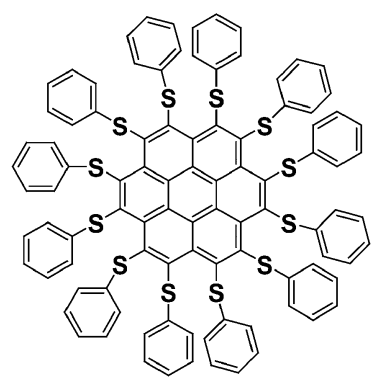

Fig. 1 The coronene core and the multidirectional electronic 3D properties of dodecakis(phenylthio)coronene (DPTC) as a polysulfurated "spider-like molecule" prompt us to further applications in dendrimer chemistry toward new molecular electronics. disordered regions around the columnar structure diffuse at RT and aggregate at the corners of the ordered columnar domains. The protruding spots of the disordered areas have an apparent diameter of approximately $1.7 \mathrm{~nm}$ corresponding to the size of a single DPTC molecule. Hence, we ascribe these spots to single DPTC molecules scattered around within the disordered regions. In the case of the $\mathrm{Au}(111)$ substrate, the strong affinity of multiple sulfide functionalities towards gold would suggest a "face-on" configuration for such a "spiderlike molecule" (coronene core parallel to the substrate). ${ }^{33}$ However, the observed highly ordered row structure is most likely described by an arrangement of the DPTC molecules with an "edge-on" orientation. From a higher resolution STM image of the columnar structure (inset of Fig. 2(a)) we obtain protruding features within each column having a distance of $a_{\mathrm{STM}}=(0.49 \pm 0.05) \mathrm{nm}$. The separation between adjacent columns is $b_{\mathrm{STM}}=(1.4 \pm 0.2) \mathrm{nm}$. Obviously, $a_{\mathrm{STM}}$ is linked to the $[11 \overline{2}]$ direction of the underlying $\mathrm{Au}(111)$ surface having a next-nearest-neighbor distance of $0.499 \mathrm{~nm}$. The alignment of the columnar stacks to the substrate lattice is further evidenced by the relative orientation of the rows in neighboring domains. Column orientations of neighboring domains enclose an angle of $120^{\circ}$ reflecting the hexagonal symmetry of the $\mathrm{Au}(111)$ surface.

\section{Supramolecular self-assembly}

For a further interpretation of the STM data, an analysis of the geometry of possible DPTC conformers would be needed. Even by simple considerations, it is clear that DPTC is not a planar molecule, even though it has a planar coronene core. Twelve phenylthio-substituents are bound to it, which are forced to adopt a conformation above or below the plane of the coronene due to the $\mathrm{C}-\mathrm{S}-\mathrm{C}$ angle of about $109^{\circ}$ and the space requirement of the phenyl rings themselves (steric effects). However, DPTC is a complex large molecule with many rotational degrees of freedom leading to many possible structural conformers. ${ }^{34}$

Regarding an "up-down" conformer coming from a release of steric hindrance, ${ }^{9}$ it is obvious that strong interactions of the core with the HOPG(0001) surface are excluded because the core is screened by the phenyl groups and cannot bind to the surface. Only weaker interactions with phenyl or thioether groups are possible. An "all-up" conformer would allow an increased interaction between the coronene core and the HOPG $(0001)$ surface. However, the distance between the coronene core and the HOPG(0001) surface would still be large due to the binding angle at the thioether groups and the space requirement of the phenyl substituents. In short, the DPTCs are most probably weakly physisorbed on HOPG(0001) due to steric hindrance.

Another columnar structure formed by "edge-on" coronene molecules was given by English and Hipps. ${ }^{35}$ In their study the coronene cores were stabilized by embedded heptanoic acid solvent molecules in between, while the supramolecular interactions in our columnar structure are mainly promoted by thiophenyl substituents.

An alternating up and down substituent pattern of DPTC in the columnar structure with an intracolumnar distance $a=2 a_{\text {STM }}$ between neighboring molecules is also supported by earlier results of X-ray crystallographic studies of persulfurated arenes. ${ }^{15}$ 
a

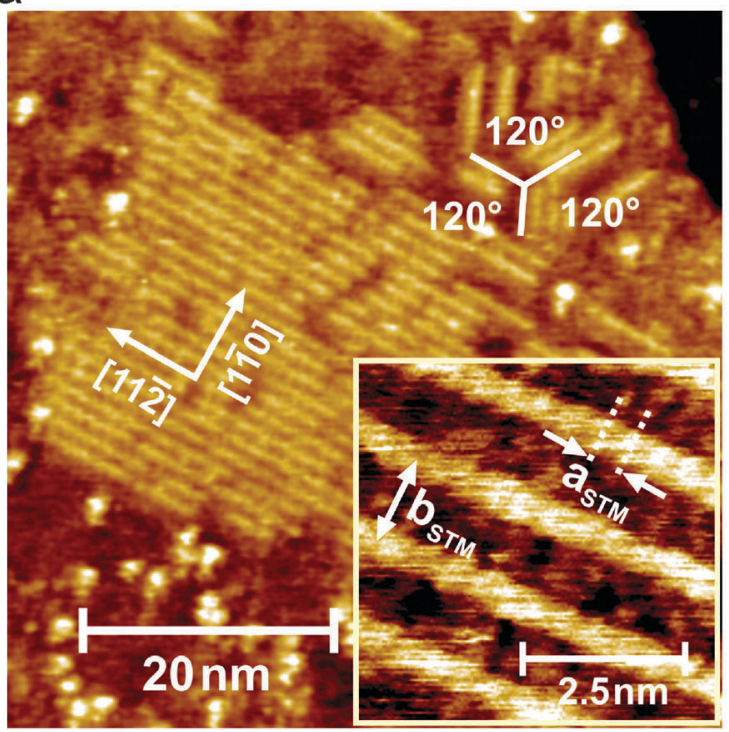

b

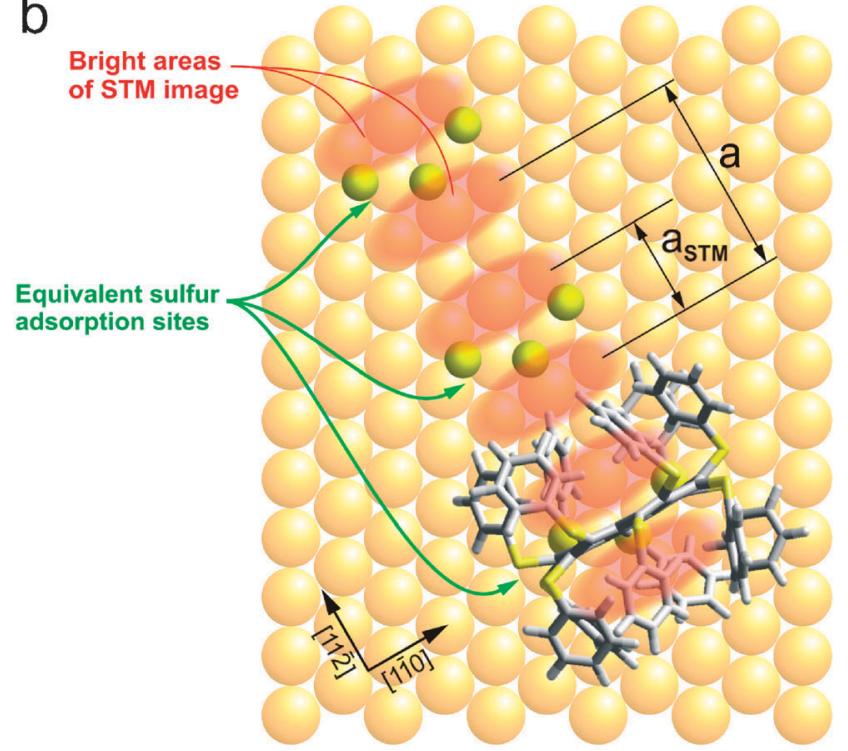

Fig. 2 (a) STM constant current topographic image ( $V=0.6 \mathrm{~V}, I=0.09 \mathrm{nA})$ of a DPTC monolayer on Au(111). DPTC molecules assemble with an "edge-on" orientation into an ordered columnar structure. Some physisorbed single DPTCs are scattered around within the disordered regions surrounding the columnar structure. Inset: higher resolution STM image ( $V=0.36 \mathrm{~V}, I=0.15 \mathrm{nA})$ of a highly ordered domain of the columnar DPTC arrangement. $a_{\mathrm{STM}}$ denotes the distance between protruding features within the columns and $b_{\mathrm{STM}}$ the intercolumnar spacing. (b) Schematic drawing showing the suggested arrangement of DPTC molecules on Au(111). Three sulfur atoms (displayed as yellow balls) of each "up-down" DPTC conformer point towards the substrate surface and can form coordinate links to the gold atoms. The columns grow along the [112] direction with the DPTCs positioned at equivalent adsorption sites. The intracolumnar distance between neighboring molecules amounts to $a=2 \sqrt{ } 3$. $a_{\mathrm{Au}(111)}$, where $a_{\mathrm{Au}(111)}$ is the lattice constant of the $\mathrm{Au}(111)$ surface. In the STM image bright areas due to protruding phenyl-groups on each side of the coronene core are observed at approximately equidistant separations of $a / 2 \approx a_{\mathrm{STM}}=(0.49 \pm 0.05) \mathrm{nm}$ (see also ESI $\ddagger$ ).

It was shown that those compounds exhibit such a conformational preference in the solid state for an up and down alternation of the substituents. For example, the smaller analogue of DPTC, having benzene instead of coronene as the central aromatic core, namely hexakis(phenylthio)benzene (HPTB), often exhibits an "up-down" conformation in the solid state. HPTB crystallizes into a triclinic unit cell of space group $P 1$ and the lattice constant along the direction perpendicular to the central benzene unit (the direction of the rows of DPTC) amounts to $c \approx 1.06 \mathrm{~nm}$. Thus, it matches with the intracolumnar spacing $a$ found for DPTC in the present study. Since the heights of the "up-down" conformers of HTPB and DPTC should be similar, comparable lattice constants are reasonable and support the supposed structural arrangement of DPTC. In addition, an approximate fit of the "up-down" conformer to the $\mathrm{Au}(111)$ would predict that three aromatic thioether groups of one DPTC could be directed simultaneously to the surface in a "edge-on" orientation (Fig. 2(b)).

\section{X-Ray photoelectron spectroscopy (XPS)}

In order to gain insights into the interactions between DPTCs and the gold surface, we performed X-ray photoelectron spectroscopy (XPS) measurements of the monolayer samples. The XPS core level spectra in the $\mathrm{S} 2 \mathrm{p}$ region (ESI $\ddagger$ ) show that the $S 2 p_{3 / 2}$ peak consists of two components. One main component at a binding energy of $163.2 \mathrm{eV}$ is related to the unbound sulfur in the thioether groups, whereas a second peak at $161.8 \mathrm{eV}$ suggests the formation of covalent bonds between gold and some of the sulfur atoms of DPTC. ${ }^{36}$ This finding additionally affirms the existence of a bond between the thioether groups of one DPTC and the gold surface according to the structural arrangement depicted in Fig. 2(b). Furthermore, the reduced intermolecular distance in the columnar stacks $\left(2 a_{\mathrm{STM}}=\right.$ width of interdigitating molecules is smaller than the width of isolated "up-down" conformer) indicates intermolecular interactions. In principle, the interaction with the $\mathrm{Au}(111)$ surface as well as intermolecular interactions should lead to a reorganization of the molecular conformation and the molecular orbitals (MOs) of the DPTCs.

\section{Fluorescence correlation spectroscopy (FCS), diffusion and aggregation studies in solution}

We would like to demonstrate that the self-organization of DPTC into columnar stacks is a real surface mediated process which takes place during the deposition on the Au(111) surface and is not due to preformed molecular stacks in the precursor solution. In order to verify that DPTC is monomolecular in solution and does not form aggregates, we determined the diffusion coefficients for DPTC in dimethylformamide (DMF) with fluorescence correlation spectroscopy (FCS) as a function of the DPTC concentration. Fig. 3(a) shows the measured FCS correlation functions for five samples with concentrations ranging from $c=7.5 \times 10^{-6} \mathrm{~mol} \mathrm{l}^{-1}$ (used for droplet deposition and UV-Vis spectroscopy) down to $c=0.8 \times 10^{-6} \mathrm{~mol}^{-1}$. The solid lines correspond to fit curves calculated by application of eqn (S1) from which the diffusion coefficients can be derived (see ESI†̣). Fig. 3(b) presents the diffusion coefficients as a function of the DPTC concentration. $D$ is found to be concentration independent yielding an average value of $\bar{D}=D_{0}=(3.2 \pm 0.2) \mathrm{m}^{2} \mathrm{~s}^{-1}$ from which by application of eqn (S2) (ESI $\ddagger$ ) the hydrodynamic 

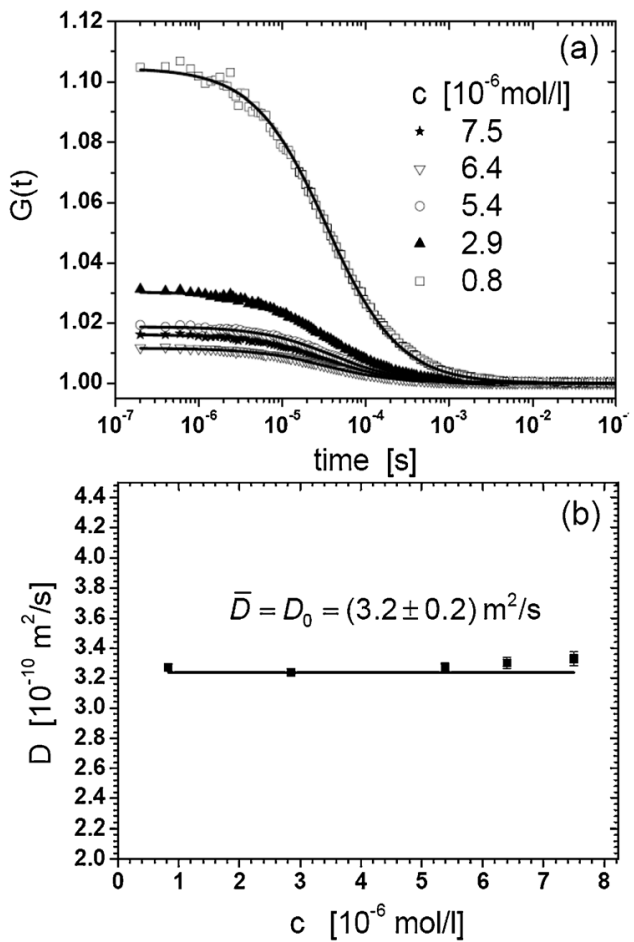

Fig. 3 (a) FCS correlation functions measured for DPTC-DMF solutions with different DPTC concentrations $c$. The solid lines correspond to a fit of the spectra with eqn (S1) (ESIț). (b) Diffusion coefficients $D$ as a function of DPTC content determined from the FCS correlation functions by application of eqn (S1) (ESI $\ddagger$ ). The solid line corresponds to the average value $D=D_{0}$.

radius $R_{\mathrm{h}}=(0.72 \pm 0.04) \mathrm{nm}$ can be calculated. The given errors include the systematic errors (around 6\%) originating from measuring against a standard. The hydrodynamic radius reflects well the size of a single molecule. In addition, we observed no dependence of the diffusion coefficient on the DPTC concentration. Consequently we can rule out aggregation at higher concentrations and significant intermolecular interactions both of which should lead to a decrease of $D$ with increasing concentration. Thus, we can conclude that DPTC is monomolecular in DMF and intermolecular interactions are not relevant up to a concentration used for the UV-Vis absorption spectroscopy and droplet deposition used for the self-assembly of monolayers. This is in agreement with previous UV-Vis results on some persulfurated coronene derivatives in $10^{-5} \mathrm{M}$ dioxane solutions, indicating that deviations from the Beer-Lambert law were negligible and therefore, intermolecular interactions were almost ruled out at such concentrations. ${ }^{14}$ Thus, the self-assembly of DPTC on Au(111) into columnar stacks builds up during the deposition process on the surface. Intermolecular $\pi-\pi$ interactions become only relevant with the decrease of competing solvating effects, when molecular conformational degrees of freedom are diminishing, and ordering is taking place on the $\mathrm{Au}(111)$ surface.

\section{Scanning tunneling spectroscopy (STS)}

Single, non-aggregated DPTC molecules (Fig. 2(a)) within the disordered regions on $\mathrm{Au}(111)$ were investigated by UHV-STS. The resulting $I(V)$ characteristic is shown in Fig. 4(a). This
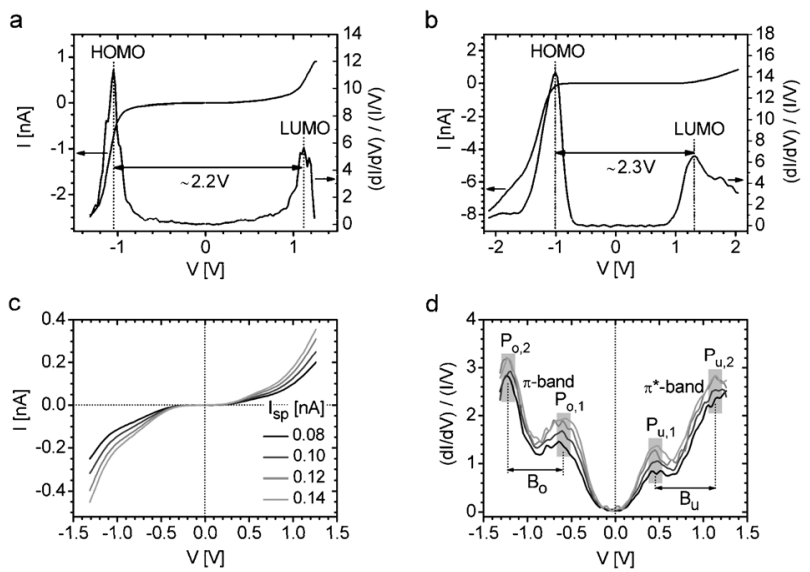

Fig. $4 I(V)$ characteristics and corresponding normalized differential conductance $(\mathrm{d} I / \mathrm{d} V) /(I / V)$ versus voltage curves obtained (a) for a non-aggregated single DPTC molecule within the disordered region on $\mathrm{Au}(111)$ (average of 20 single $I(V)$ traces with $V_{\mathrm{SP}}=0.9 \mathrm{~V}, I_{\mathrm{SP}}=0.12 \mathrm{nA}$, $T=300 \mathrm{~K}$ ) and (b) on the approximately one monolayer DPTC covered HOPG $(0001)$ surface (average of 100 single $I(V)$ traces with $\left.V_{\mathrm{SP}}=1.45 \mathrm{~V}, I_{\mathrm{SP}}=0.17 \mathrm{nA}, T=77 \mathrm{~K}\right)$. See also Fig. S1 in the ESI + for the monolayer structure of DPTC at $77 \mathrm{~K}$. In both cases, the peaks in the normalized differential conductance are ascribed to a resonant charge transport through mainly undisturbed HOMO and LUMO molecular states. (c) $I(V)$ curves and (d) normalized differential conductance versus voltage curves of stacked DPTC molecules within the area of the columnar "edge-on" arrangement on $\mathrm{Au}(111)$. The peaks in the $(\mathrm{d} I / \mathrm{d} V) /(I / V)$ curves are attributed to the increased density of states occurring at the edges of the expected one-dimensional bands formed by stacking interactions mainly along the direction of the columns. The reproducible occurrence of relevant density of states features was controlled by measuring $I(V)$ curves with different values for $I_{\mathrm{SP}}$, i.e. varying tip-sample distances, while keeping $V_{\mathrm{SP}}=0.9 \mathrm{~V}$ constant. Each of the displayed curves represents an average over 100 single $I(V)$ traces measured at $T=300 \mathrm{~K}$.

curve shows a distinct tunneling gap (nearly zero conductance) within a given energetic window and a sharp rise in current above certain threshold voltages within the negative and positive bias voltage region. The pronounced peaks in the normalized differential conductance $(\mathrm{d} I / \mathrm{d} V) /(I / V)$ are assigned to tunneling through the HOMO and LUMO states, respectively. The distance between these resonances, i.e. the HOMO-LUMO gap, is measured to be $\Delta E_{\mathrm{STS}} \approx 2.2 \mathrm{eV}$.

For comparison, we measured the UV-Vis absorption spectrum of DPTC in solution. From the onset of the energetically lowest absorption band, we determined an optical HOMOLUMO gap of $\Delta E_{\text {OPT }}(2.05 \pm 0.05) \mathrm{eV}$. The optical gap corresponds to the exciton transition energy. Compared to STS measurements it is lowered by the exciton binding energy which is expected to be in the range of a few 100 $\mathrm{meV}$ for large $\pi$-conjugated molecules. ${ }^{37}$ Thus, a gap of $2.2 \mathrm{eV}$ obtained with STS seems to be a reasonable result for a single molecule having weak interactions with the surrounding molecules and the metal substrate. This small HOMO-LUMO gap of DPTC, in comparison with other aromatic molecules, also points to an extremely large conjugated system resulting in a thorough delocalization of the states. The HOMOLUMO gaps decrease according to the increasing size of the $\pi$-conjugated system, i.e. the value of the HOMO-LUMO gap 
for phenylenes is $\sim 5 \mathrm{eV},{ }^{38}$ for pentacene $4.1 \mathrm{eV}^{39}$ and for hexa-peri-hexabenzocoronene $3.2 \mathrm{eV}(2.8 \mathrm{eV}$ as measured by UV-Vis absorption). ${ }^{40}$ Our results are in agreement with cyclic voltammetry measurements which demonstrated that the phenylthio-substitution of the central aromatic core leads to an effective delocalization of charge throughout the molecular system. $^{13}$

For DPTC deposited on the HOPG(0001) surface, we obtained an orbital mediated tunneling through HOMO and LUMO molecular states which is similar to the measured characteristics for the single, non-aggregated molecules on $\mathrm{Au}(111)$. Fig. 4(b) shows the $I(V)$ characteristic and the normalized differential conductance for DPTC molecules adsorbed on HOPG(0001) at $T=77 \mathrm{~K}$. The distinct peaks can again be assigned to tunneling via the frontier molecular orbitals. The measured HOMO-LUMO gap amounts to $\Delta E_{\mathrm{STS}} \approx 2.3 \mathrm{eV}$, which is comparable to the value obtained for the single DPTC molecules on $\mathrm{Au}(111)$. This suggests that DPTCs within the adsorbate layer on HOPG(0001) have weak electronic interactions with adjacent molecules or the substrate. Note that the spectrum of Fig. 4(b) is an average of 100 single curves. Individual $I(V)$ traces (ESI $\ddagger$ ) show variations in the onset of the orbital mediated tunneling process. Such variations of the energetic positions of the molecular resonances are rather self-evident when considering the disordered nature of the molecular layer and the fact that the molecules might not be fully immobilized even at $T=77 \mathrm{~K}$. Therefore, the molecule/substrate interface and therewith the electrostatic potential profile are not welldefined. Also, the distance to neighboring molecules and consequently the polarization contributions may vary for different $I(V)$ traces.

Fig. 4(c) shows the $I(V)$ characteristics obtained in the area of the columnar "edge-on" arrangement of DPTC. The corresponding normalized differential conductance curves (Fig. 4(d)) reveal the strong influence of the columnar stacking on the electronic structure. The curves show that a band of states forms. This suggests that electronic interaction exists between the wave functions of the stacked molecules which leads to a splitting of the energy levels into stabilized and destabilized ones. ${ }^{4-44}$ Furthermore, it is expected that electronic rearrangements occur due to interactions of the "edge-on" DPTCs with the surface metal atoms, i.e. due to the coordinate links between the thioether groups of the DPTCs and the gold atoms. A first estimation of the interaction strength between adjacent molecules within the columns can be obtained by considering a mainly one-dimensional dispersion, i.e. charge can be much more easily transferred between adjacent molecules of one column than between neighboring columns. Within the tight-binding model including nearest-neighbor interactions the dispersion relation can be written as $E_{\mathrm{o}, \mathrm{u}}(k)=\varepsilon_{\mathrm{o}, \mathrm{u}}+$ $2 t_{\mathrm{o}, \mathrm{u}} \cos (k a)$. Here, the subscripts o and $\mathrm{u}$ denote the band of occupied states ( $\pi$-band) and unoccupied states $\left(\pi^{*}\right.$-band), respectively, $\varepsilon_{\mathrm{o}, \mathrm{u}}$ are the centers of each band, $t_{\mathrm{o}, \mathrm{u}}$ denotes the transfer integrals in the directions of the columns, $a$ is the lattice constant and $k$ is the reciprocal lattice vector. ${ }^{41,42}$ The transfer integral expresses the strength of the electronic coupling of the molecular orbitals between the adjacent molecules. For a finite number $n$ of interacting molecules, the discrete values of $k$ can be written as $k=m \pi /[a(n+1)]$, with $1 \leq m \leq n$. For the presented tunneling spectra the number of DPTC molecules in a stack is approximately $n \approx 40$. The peaks in the normalized differential conductance can approximately be assigned to the increased density of states occurring at the band edges. Thus, the transfer integrals are estimated to be $\left|t_{\mathrm{o}, \mathrm{u}}\right| \approx B_{\mathrm{o}, \mathrm{u}} / 4$, where $B_{\mathrm{o}, \mathrm{u}}$ is the experimentally derived bandwidth (see Fig. 4(d)). The obtained values $t_{\mathrm{u}} \approx 0.17 \mathrm{eV}$ and $t_{\mathrm{o}} \approx 0.16 \mathrm{eV}$ are within the range of the expected transfer integrals of $\pi$-stacked PAHs. ${ }^{42,45}$ Thus, interactions of MOs of adjacent molecules are assumed to be the predominant factor for band formation in the case of $\pi-\pi$ stacking motif of planar PAH systems. The polyaromatic core is responsible for the intermolecular electronic overlap. However, the band formation from the DPTC stacks originates from the interdigitation of the peripheral phenyl groups.

The role of aryl-sulfide groups is thus dominant. Since electrons are delocalized throughout the whole molecular system $^{14}$ in isolated DPTC molecules, the overlap between the peripheral groups might enable a charge delocalization throughout the columnar stacks. Nevertheless, a detailed understanding has to include the electronic modifications introduced by molecule-metal and intermolecular interactions. This will be a challenging task due to the complex structures, but it is in progress while employing DFT calculations.

\section{Conclusions}

Dodecakis(phenylthio)coronene molecules (DPTCs) are part of a family of persulfurated aromatic compounds, which were not well-studied on metal or graphite surfaces, in spite of numerous and rich supramolecular interactions involving a high density of aromatic rings and sulfide linkages. We demonstrated that they weakly physisorb on HOPG(0001). By comparison to other persulfurated benzene structures and the HOMO-LUMO gap experimentally obtained, it is hypothesized that adsorption occurs in the most stable "up-down" conformation on HOPG. This conformer would prevent direct interactions between the coronene core and the HOPG(0001) surface due to steric hindrance and thus only weak physisorption is possible. The HOMO-LUMO gap we deduced for single DPTC molecules, which is also applicable for molecules on HOPG, amounts to $\Delta E_{\mathrm{STS}} \approx 2.2 \mathrm{eV}$. This value corresponds to a large conjugated system with an effective delocalization of charge throughout the molecular system, in agreement with UV-Vis and cyclic voltammetry results. $^{14}$

Furthermore, single DPTC molecules and DPTC columns aligned to the substrate lattice were identified on $\mathrm{Au}(111)$. Since no preassembly in solution has been observed, as confirmed by fluorescence correlation spectroscopy (FCS), this columnar assembly is assumed to be a surface-mediated process. The highly ordered row structure is best hypothesized by a sequential stacking of "up-down" conformers in an "edge-on" orientation (coronene core perpendicular to the $\mathrm{Au}(111)$ surface). Surface-molecule interactions are facilitated by some supramolecular interactions of many aryl-sulfide groups and the $\mathrm{Au}(111)$ surface. The tunneling spectra of those highly ordered stacks show an energetic splitting of the 
molecular states, which can be interpreted in terms of an intermolecular stacking motif of three-dimensional aromatic molecules which enables the build-up of $1 \mathrm{D}$ electron channels via interdigitation of aryl-sulfide groups.

As a general conclusion, aryl-sulfide groups provide the most determinant electronic and physical properties of the coronene molecular asterisk DPTC. This novel observation might lead to further applications in the chemistry of dendrimers, while using the coronene core properties and its multidirectional electronic properties toward novel molecular electronics. It could also serve as a model for a better understanding of supramolecular and substrate-molecule interactions. Future work is in progress in that direction.

\section{Experimental section}

\section{Synthesis of DPTC}

The synthesis of dodecakis(phenylthio)coronene (DPTC) is based on the sulfuration of dodecachlorocoronene and follows a similar procedure as previously described for dodecakis (4-methyl-1-phenylthio)coronene. ${ }^{13}$ The preparation of dodecachlorocoronene in a quantitative yield was based on a previous protocol reporting the perchlorination of coronene. ${ }^{25}$ Into a dry two-necked flask under nitrogen were introduced thiophenol (352 mg, $3.10 \mathrm{mmol}$ ) and dodecachlorocoronene (168 mg, $0.24 \mathrm{mmol})$, followed by a syringe addition of dry 1,3dimethyl-2-imidazolidinone (DMI) $(5.0 \mathrm{~mL}$, dried over activated $3 \AA$ molecular sieves). After cooling the mixture in an ice-bath at $3{ }^{\circ} \mathrm{C}$, powdered $\mathrm{NaH} 95 \%(140 \mathrm{mg} ; 6.6 \mathrm{mmol})$ was slowly added in small portions via a lateral tube (Gooch tube) while stirring vigorously until the foam disappeared. The resulting mixture turned deep red over time at $20{ }^{\circ} \mathrm{C}$ and stirring was continued for 2 hours. After adding an aqueous solution of $\mathrm{NaOH}(1 \mathrm{M}, 10 \mathrm{~mL})$, an extraction was performed with DCM $(2 \times 30 \mathrm{~mL})$. The collected organic phases were washed with $\mathrm{H}_{2} \mathrm{O}(3 \times 100 \mathrm{~mL})$, and dried over anhydrous $\mathrm{Na}_{2} \mathrm{SO}_{4}$. Filtration and evaporation of the solvent led to the crude product (still containing traces of DMI). A first trituration in EtOH $(5 \mathrm{~mL})$ while stirring vigorously and a filtration were achieved. A final trituration led to a pure product after drying under high vacuum (260 mg; 69\%, deep red powder); m.p. $340{ }^{\circ} \mathrm{C}$ (dec.); $R_{\mathrm{f}}=0.24\left(\mathrm{SiO}_{2}\right.$, petroleum ether/DCM: 60/40); ${ }^{1} \mathrm{H}$ NMR (200 MHz, CDCl 3 , TMS, $\delta$ ppm): $6.55\left(d_{\text {app }}, J=\right.$ $7.2 \mathrm{~Hz}, 24 \mathrm{H}, \mathrm{Ar}-\mathrm{H}$ ortho); 6.94-7.03 (m, 36H, Ar-H metal para); ${ }^{13} \mathrm{C}$ NMR (63 MHz, $\left.\mathrm{CDCl}_{3}, \mathrm{TMS}, \delta \mathrm{ppm}\right): 128.47$ $(\mathrm{CH}), 126.64(\mathrm{CH}), 125.25(\mathrm{CH}), 120.90(\mathrm{C}-\mathrm{S}$ phenyl); UV-vis ( $n$-hexane): $\lambda_{\max }(\varepsilon)=262 \mathrm{~nm}(175500) ; 434 \mathrm{~nm}(125000)$; MALDI-TOF MS (DCTB, AgTFA, THF, $\lambda 337 \mathrm{~nm}$ ); $m / z$ for $\mathrm{C}_{96} \mathrm{H}_{60} \mathrm{~S}_{12}\left[\mathrm{M}^{+}\right.$.]: 1596.13; found: 1596.13 . Cyclic voltammetry: two reversible one-electron reduction waves $\left(v s . \mathrm{Fc} / \mathrm{Fc}^{+}\right)$: $E_{1}^{0}=-1.42 \mathrm{~V} ; E_{2}^{0}=-1.83 \mathrm{~V}$.

\section{Scanning tunneling microscopy (STM) and spectroscopy (STS)}

Those measurements were performed at room temperature or $77 \mathrm{~K}$ under UHV conditions (pressure $p \approx 1 \times 10^{-10} \mathrm{mbar}$ ) using a JEOL 4500S STM head with electrochemically etched tungsten tips. As substrates we used (1) highly oriented pyrolytic graphite $\left(\mathrm{HOPG}\right.$, mosaic angle $\left.0.4^{\circ}\right)$ freshly cleaved along its (0001) plane and (2) $\mathrm{Au}(111)$ thin films which were prepared on mica in a two step deposition process. ${ }^{26}$ The conditions to self-assemble DPTC on HOPG and Au(111) were optimized by testing solvents (toluene, dimethylformamide, and 1,2,4-trichlorobenzene), the molar concentrations $\left(10^{-4}\right.$ to $10^{-6} \mathrm{~mol} \mathrm{l}^{-1}$ ), and self-assembly times from 1 minute to 12 hours. Monolayers of DPTC were obtained from a diluted DPTC-solution ( $7 \mu$ solution was used for a sample of about $20 \mathrm{~mm}^{2}$, concentration $7.5 \times 10^{-6} \mathrm{~mol}^{-1}$ ). 1,2,4-Trichlorobenzene and toluene were used as solvents for deposition on HOPG and Au(111), respectively. The concentrations of the solutions were chosen such that the substrates were covered approximately with one monolayer of molecules, assuming a closed packing of DPTCs with coronene core parallel to the substrate surface. After complete evaporation, the dry sample was immediately transferred into the UHV-STM/STS measurement chamber. Current-voltage curves, $I(V)$, were recorded with 128 equidistant bias voltage steps after switching off the feedback loop. The tip-sample distance was adjusted according to the established current set point $I_{\mathrm{SP}}$ at a given voltage set point, $V_{\text {SP. }}$ The normalized differential conductance, $(\mathrm{d} I / \mathrm{d} V) /$ $(I / V)$, which can be related to the density of states (DOS) at the sample surface, was calculated numerically from the measured $I(V)$ curves. $^{27-29}$

\section{UV-Vis absorption spectroscopy}

The absorption spectrum of DPTC in dimethylformamide (DMF) $\left(c=7.5 \times 10^{-6} \mathrm{~mol}^{-1}\right)$ was measured with a Kontron Uvikon 860 double-beam spectrophotometer. The spectrum was corrected for absorption occurring in the solvent and the quartz cuvette.

\section{Fluorescence correlation spectroscopy (FCS)}

The fluorescence intensity correlation function ${ }^{30,31}$ was measured with a commercial confocal system using an Axiovert $200 \mathrm{M}$ microscope Confocor 2 (Carl Zeiss GmbH, Germany) with a $40 \times$ Plan Neofluar multiimmersion objective (numerical aperture of 0.9). For excitation the $488 \mathrm{~nm}$ line of a $25 \mathrm{~mW}$ argon laser was used. In order to avoid artificial contributions to the signal from detector afterpulsing the pseudo-cross correlation was measured. Measurements were performed at room temperature $\left(T=20{ }^{\circ} \mathrm{C}\right)$ using well-sealed, steel sample containers with a cover glass (thickness $0.17 \mathrm{~mm}$ ) at the bottom. The highest concentration measured corresponds to a DPTC-DMF solution with a DPTC content of $c=$ $7.5 \times 10^{-6} \mathrm{~mol} \mathrm{l}^{-1}$ as used for droplet deposition and UV-Vis spectroscopy. Lower concentrations were prepared by dilution of the stock solution.

\section{Acknowledgements}

M.G. thanks the French National Center for Scientific Research (CNRS) and Aix-Marseille University (Université de la Méditerranée), as well as Dr C. Dallaire and Dr A. Pinchart for many synthetic investigations on coronene derivatives at ULB in Brussels. M.G. also acknowledges the Spectropole of Marseille. P.K. and S.K. thank N. Atodiresei, V. Caciuc and S. Blügel for fruitful discussions and also H. Haselier and H. John for their assistance. 


\section{Notes and references}

1 G. Maruccio, R. Cingolani and R. Rinaldi, J. Mater. Chem., 2004, 14, 542-554.

2 A. Nitzan and M. A. Ratner, Science, 2003, 300, 1384-1389.

3 N. J. Tao, Nat. Nanotechnol., 2006, 1, 173-181.

4 S. De Feyter and F. C. De Schryver, Chem. Soc. Rev., 2003, 32, 139-150.

5 C. D. Simpson, J. Wu, M. D. Watson and K. Müllen, J. Mater. Chem., 2004, 14, 494-504.

6 S. Sergeyev, W. Pisula and Y. H. Geerts, Chem. Soc. Rev., 2007, 36, 1902-1929.

7 D. Adam, P. Schuhmacher, J. Simmerer, L. Häussling, K. Siemensmeyer, K. H. Etzbach, H. Ringsdorf and D. Haarer, Nature, 1994, 371, 141-143.

8 A. M. van de Craats, J. M. Warman, A. Fechtenkötter, J. D. Brand, M. A. Harbison and K. Müllen, Adv. Mater., 1999, 11, 1469-1472.

9 P. Kowalzik, N. Atodiresei, M. Gingras, V. Caciuc, S. Blügel, R. Waser and S. Karthäuser, J. Chem. Phys. C, 2011, 115, 9204-9209.

10 (a) R. Adams, W. Reifschneider and M. D. Nair, Croat. Chim. Acta, 1957, 29, 277-285; (b) R. Adams and A. Ferretti, J. Am. Chem. Soc., 1959, 81, 4927-4931.

11 S. C. Jensen, A. E. Baber, H. L. Tierney and E. C. H. Sykes, ACS Nano, 2007, 1, 423-428 and references therein.

12 E. B. Troughton, C. D. Bain, G. M. Whitesides, R. G. Nuzzo, D. L. Allara and M. D. Porter, Langmuir, 1988, 4, 365-385.

13 J. H. R. Tucker, M. Gingras, H. Brand and J.-M. Lehn, J. Chem. Soc., Perkin Trans. 2, 1997, 1303-1307.

14 M. Gingras, A. Pinchart, C. Dallaire, T. Mallah and E. Levillain, Chem--Eur. J., 2004, 10, 2895-2904.

15 M. Gingras, J.-M. Raimundo and Y. M. Chabre, Angew. Chem., Int. Ed., 2006, 45, 1686-1712.

16 M. Gingras, A. Pinchart and C. Dallaire, Angew. Chem., Int. Ed., 1998, 37, 3149-3151.

17 R. Temirov, S. Soubatch, A. Luican and F. S. Tautz, Nature, 2006, 444, 350-253.

18 N. Gonzalez-Lakunza, I. Fernández-Torrente, K. J. Franke, N. Lorente, A. Arnau and J. I. Pascual, Phys. Rev. Lett., 2008, 100, 156805-156809.

19 A. Rochefort, R. Martel and P. Avouris, Nano Lett., 2002, 2, 877-880.

20 D. S. Seferos, S. A. Trammell, G. C. Bazan and J. G. Kushmerick, Proc. Natl. Acad. Sci. U. S. A., 2005, 102, 8821-8825.

21 R. Foster, in Organic Charge-Transfer Complexes, Academic Press, London, 1969.

22 A. Gesquière, S. De Feyter, F. C. De Schryver, F. Schoonbeek, J. van Esch, R. M. Kellogg and B. L. Feringa, Nano Lett., 2001, 1, 201-206.

23 H. Uji-i, S. Nishio and H. Fukumura, Chem. Phys. Lett., 2005, 408, 112-117.

24 F. Jäckel, M. D. Watson, K. Müllen and J. P. Rabe, Phys. Rev. B: Condens. Matter, 2006, 73, 045423.
25 T. Baird, J. H. Gall, D. D. MacNicol, P. R. Mallinson and C. R. Michie, J. Chem. Soc., Chem. Commun., 1988, 1471-1473 and references therein.

26 B. Lüssem, S. Karthäuser, H. Haselier and R. Waser, Appl. Surf. Sci., 2005, 249, 197-202.

27 R. M. Feenstra, J. A. Stroscio and A. P. Fein, Surf. Sci., 1987, 181, 295-306.

28 M. Toerker, T. Fritz, H. Proehl, R. Gutierrez, F. Großmann and R. Schmidt, Phys. Rev. B: Condens. Matter, 2002, 65, 245422.

29 P. G. Collins, J. C. Grossman, M. Côté, M. Ishigami, C. Piskoti, S. G. Louie, M. L. Cohen and A. Zettl, Phys. Rev. Lett., 1999, 82, 165-168.

30 S. Rathgeber, H.-J. Beauvisage, H. Chevreau, N. Willenbacher and C. Oelschlaeger, Langmuir, 2009, 25, 6368-6376.

31 O. Krichevsky and G. Bonnet, Rep. Prog. Phys., 2002, 65, 251-297.

32 S. De Feyter and F. C. De Schryver, Top. Curr. Chem., 2005, 258, 205-255 and references therein.

33 H. Schönherr, F. J. B. Kremer, S. Kumar, J. A. Rego, H. Wolf, H. Ringsdorf, M. Jaschke, H.-J. Butt and E. Bamberg, J. Am. Chem. Soc., 1996, 118, 13051-13057.

34 A preliminary work on optimized geometries and HOMO-LUMO orbitals of DPTC conformers using ab initio calculations was undertaken by N. Atodiresei, V. Caciuc and S. Blügel. Due to the complexity, a full account will be published elsewhere. DPTC conformers considered here: (a) "up-down" conformer, 12 phenyl rings are situated by alternation above and below the plane of the coronene unit (width: $1.31 \mathrm{~nm}$, height: $1.19 \mathrm{~nm}$ ); (b) "up-up" conformer, all 12 phenyl rings are located above the plane of the coronene unit (width: $1.94 \mathrm{~nm}$, height: $0.71 \mathrm{~nm}$ ).

35 W. A. English and K. W. Hipps, J. Phys. Chem. C, 2008, 112 2026-2031.

36 T. Ishida, N. Choi, W. Mizutani, H. Tokumoto, I. Kojima, H. Azehara, H. Hokari, U. Akiba and M. Fujihira, Langmuir, 1999, 15, 6799-6806.

37 M. Knupfer, J. Fink, E. Zojer, G. Leising and D. Fichou, Chem. Phys. Lett., 2000, 318, 585-589.

38 A. Salomon, D. Cahen, S. Lindsay, J. Tomfohr, V. Engelkes and D. Frisbie, Adv. Mater., 2003, 15, 1881-1890.

39 J. Repp, G. Meyer, S. Paavilainen, F. Olsson and M. Persson, Science, 2006, 312, 1196-1199.

40 H. Proehl, M. Toerker, F. Sellam, T. Fritz, K. Leo, C. Simpson and K. Müllen, Phys. Rev. B: Condens. Matter, 2001, 63, 205409.

41 J.-L. Brédas, J. P. Calbert, D. A. da Silva Filho and J. Cornil, Proc. Natl. Acad. Sci. U. S. A., 2002, 99, 5804-5809.

42 J. Cornil, V. Lemaur, J.-P. Calbert and J.-L. Brédas, Adv. Mater., 2002, 14, 726-729.

43 J. Huang and M. Kertesz, J. Chem. Phys., 2005, 122, 234707.

44 N. Ueno and S. Kera, Prog. Surf. Sci., 2008, 83, 490-557.

45 X. Feng, V. Marcon, W. Pisula, M. R. Hansen, J. Kirkpatrick, F. Grozema, D. Andrienko, K. Kremer and K. Müllen, Nat. Mater., 2009, 8, 421-426. 